\title{
The Impact of Combinations of Alcohol, Nicotine, and Cannabis on Dynamic Brain Connectivity
}

\author{
Victor M Vergara ${ }^{*, 1}$, Barbara J Weiland ${ }^{2}$, Kent E Hutchison ${ }^{2}$ and Vince D Calhoun' \\ 'The Mind Research Network and Lovelace Biomedical and Environmental Research Institute, Albuquerque, NM, USA; ${ }^{2}$ Departments of \\ Psychology and Neuroscience, University of Colorado, Boulder, CO, USA
}

\begin{abstract}
Alcohol, nicotine, and cannabis are among the most commonly used drugs. A prolonged and combined use of these substances can alter normal brain wiring in different ways depending on the consumed cocktail mixture. Brain connectivity alterations and their change with time can be assessed using functional magnetic resonance imaging ( $(M \mathrm{RI})$ because of its spatial and temporal content. Here, we estimated dynamic functional network connectivity (dFNC) as derived from $\mathrm{fMRI}$ data to investigate the effects of single or combined use of alcohol, nicotine, and cannabis. Data from 534 samples were grouped according to their substance use combination as controls (CTR), smokers (SMK), drinkers (DRN), smoking-and-drinking subjects (SAD), marijuana users (MAR), smoking-and-marijuana users (SAM), marijuana-anddrinking users (MAD), and users of all three substances (ALL). The DRN group tends to exhibit decreased connectivity mainly in areas of sensorial and motor control, a result supported by the dFNC outcome and the alcohol use disorder identification test. This trend dominated the SAD group and in a weaker manner MAD and ALL. Nicotine consumers were characterized by an increment of connectivity between dorsal striatum and sensorimotor areas. Where possible, common and separate effects were identified and characterized by the analysis of dFNC data. Results also suggest that a combination of cannabis and nicotine have more contrasting effects on the brain than a single use of any of these substances. On the other hand, marijuana and alcohol might follow an additive effect trend. We concluded that all of the substances have an impact on brain connectivity, but the effect differs depending on the dFNC state analyzed. Neuropsychopharmacology (2018) 43, 877-890; doi:I0.1038/npp.2017.280; published online 20 December 2017
\end{abstract}

\section{INTRODUCTION}

The 2014 global drug survey indicates that alcohol, nicotine, and cannabis are the three most used drugs (Winstock, 2014). Prolonged use of these substances may end in addiction, a disease surrounded by behavioral and socialcontext aspects influenced by biological changes in the brain (Doyon et al, 2013; Leshner, 1997; Volkow et al, 2014). Several theories have emerged as an attempt to explain the underlying mechanism of addiction. One of these views is the anhedonia hypothesis that the subjective pleasure induced by dopamine concentrations in the brain plays a critical role in reinforcement, motivation, and reward (Wise, 2008,2010). Although drugs of abuse alter dopamine concentrations in limbic brain regions, these dopaminergic alterations are not sufficient to explain the whole process of addiction (Goldstein and Volkow, 2002). For example, transitioning from voluntary to habitual drug use may be a consequence of impaired brain areas involved in executive control over behavioral inhibition processes (Everitt and Robbins, 2005; Goldstein and Volkow, 2002). Furthermore,

\footnotetext{
*Correspondence: Dr VM Vergara, The Mind Research Network, Lovelace Biomedical and Environmental Research Institute, I I0I Yale Boulevard, Albuquerque, NM 87I06, USA, Tel: + I 505272 5028, Fax: + I 505272 8002, E-mail: vvergara@mm.org

Received I 5 May 2017; revised 7 October 2017; accepted 2 November 2017; accepted article preview online 14 November 2017
}

alterations in the mesolimbic dopamine system caused by addictive substances may be the starting point of a series of neuroadaptations that produces changes in a neurocircuitry composed of the prefrontal cortex, cingulate, amygdala, insula, and the striatum (Koob and Volkow, 2010). These observations promote an increasing interest in characterizing interactions among components of the proposed neurocircuitry along with the impact it might have in other brain areas. The emergence of neuroimaging technologies facilitates the study of these interactions by providing information regarding connections among brain areas. Brain connectivity allows for the study of an important aspect based on brain circuitry and provides additional ways to look at the complex mechanism of addiction (Pariyadath et al, 2016; Sutherland et al, 2012).

Substances of abuse act as modifiers of the dopaminereward systems that affect the nucleus accumbens (NAc) and ventral tegmental area (VTA), but through different molecular mechanisms (Nestler, 2005). Pertinent literature suggests that these differences translate into diverse effects in functional connectivity. Alcohol produces dysfunctions in connectivity between the NAc and other cortical areas, including those with increased activation during stimuli and response demanding tasks (Camchong et al, 2013c). Changes in the functional connectivity of cognitive, motor, and coordination brain areas have also been linked to alcohol use (Camchong et al, 2013b; Chanraud et al, 2011). Nicotine 
binding to nicotinic acetylcholine receptors (nAChR) changes dopaminergic signaling in the areas of the reward pathway such as VTA and NAc (Subramaniyan and Dani, 2015). However, the effect of nicotine in functional connectivity is more prominent in areas like the insula (Sutherland et al, 2012). Furthermore, nicotine administration might increase functional connectivity in motor, attention, and memory brain areas (Jasinska et al, 2014). In addition to the reward system, cannabis also produces alterations of functional connectivity that include the orbitofrontal cortex (Filbey et al, 2014), default mode network, and insula (Pujol et al, 2014). Evidence supports the idea that combined substance use has different effects from that of single substance use. The use of both nicotine and marijuana enhances $\mathrm{nAChR}$ availability in the prefrontal cortex and the thalamus to a higher degree than single substance use (Brody et al, 2016). Reports indicate that combined use can also increase connectivity disruptions in posterior cortical and frontoparietal regions (Jacobsen et al, 2007). Combined alcohol and nicotine consumption also produces neurologic consequences different from single substance use (Meyerhoff et al, 2006). One important effect is that alcohol consumers are sensitive to the cognitive enhancement effects of nicotine (Ceballos et al, 2006).

This work utilizes dynamic functional network connectivity (dFNC) and multivariate approaches to investigate the different effects of alcohol, nicotine, and/or cannabis. In dFNC, connectivity is estimated at relatively short time lengths. A small set of connectivity patterns, termed dFNC states, appear successively through time (Allen et al, 2014). Because of its fine time resolution, a dFNC analysis results in a high dimensional data set. Given the large amount of dFNC variables influenced by substance comorbidity and important confounding problems (Meyerhoff et al, 2006), we selected a multivariate approach as appropriate for dFNC data and poly-substance studies (Richmond-Rakerd et al, 2016). Although the nature of this dFNC study is mainly exploratory, we expect to find previously unobserved effects or confirmatory evidence for outcomes formerly observed in functional connectivity. We also expect a disentanglement of comorbid effects as aided by the fine timescale in dFNC analysis.

\section{MATERIALS AND METHODS}

\section{Subjects}

The sample cohort included 534 subjects (195 females) between the ages of 18 and $55(33.2 \pm 9.7)$ years. Subject exclusion criteria included injury to the brain, brain-related medical problems, and bipolar or psychotic disorders. A urinalysis test confirmed or rejected the use of drugs including marijuana. The severity of alcohol use was assessed by applying the Alcohol Use Disorder Identification Test (AUDIT) (Saunders et al, 1993). AUDIT scores up to seven suggested abstinence and AUDIT scores eight or larger characterized drinking status (Vergara et al, 2017b; Weiland et al, 2014). Nicotine dependence levels were assessed using the Fagerstrom Tolerance Questionnaire (FTQ) (Fagerström, 1978). Smoking was avoided $3 \mathrm{~h}$ before scanning. Subjects with FTQ scores of $\geqslant 7$ were cataloged as smokers (Moore et al, 1987). Measurements of timeline follow-back (TLFB) approach determined the level of marijuana use (60TLFB Marijuana Days). A 60TLFB of $\geqslant 15$ days defined marijuana use status. The combinations of thresholded substance use and dependence scores were used to define eight subject groups: controls (CTR), smoking (SMK), drinking (DRN), smoking-and-drinking (SAD), marijuana (MAR), marijuana-and-drinking (MAD), smoking-and-marijuana (SAM), and users of all three substances (ALL). The CTR group did not include AUDIT scores, but did not show alcohol abuse/dependence as assessed using the Structured Clinical Interview for DSM-IV-TR Axis I Disorders, Research Version, Patient Edition (SCID-I/P) (First et al, 2002). All control subjects reported no use of nicotine or marijuana. Other relevant data collected included the Beck Depression Inventory (BDI) (Beck et al, 1988), the Beck Anxiety Inventory (Leyfer et al, 2006), the Impulsive Sensation Seeking Scale (ImpSS) (Zuckerman, 1996), and income was used as a socioeconomic status variable. Income was categorized in 7 levels: 1: \$0-\$9999/year, 2: \$10000\$19 999/year, 3: \$20 000-\$29 999/year, 4: \$30 000-\$39 999/ year, 5: \$40 000-\$49 999/year, 6: \$50 000-\$59 999/year, and 7: $>\$ 60,000 /$ year. Table 1 shows detailed information about the eight groups.

\section{MRI Data}

Data were collected on a $3 \mathrm{~T}$ Siemens TIM Trio (Erlangen, Germany) scanner. Echo-planar EPI sequence images $\left(\mathrm{TR}=2000 \mathrm{~ms}, \mathrm{TE}=29 \mathrm{~ms}\right.$, flip angle $\left.=75^{\circ}\right)$ were acquired with an 8-channel head coil. Volumes consisted of 33 axial slices $\left(64 \times 64\right.$ matrix, $3.75 \times 3.75 \mathrm{~mm}^{2}, 3.5 \mathrm{~mm}$ thickness, $1 \mathrm{~mm}$ gap). Data were preprocessed using the statistical parametric mapping software (SPM; http://www.fil.ion.ucl. ac.uk/spm) (Friston, 2003), including slice-timing correction, realignment, co-registration, spatial normalization, and transformation to the Montreal Neurological Institute (MNI) standard space. Realignment parameters were regressed out of the functional magnetic resonance imaging (fMRI) data and then smoothed using a FWHM Gaussian kernel of size $6 \mathrm{~mm}$. Group ICA (Calhoun et al, 2001; Calhoun and Adali, 2012), available through the Group ICA fMRI Toolbox (GIFT; http://mialab.mrn.org/software/gift/), was then applied to obtain a set of 100 independent components. A total of 39 out of the 100 estimated components were selected based on frequency content and visual inspection (Allen et al, 2011). In this analysis, a component is also named a resting state network (RSN) as it comprised a network including several areas of the brain and the fMRI scan was obtained during resting state. The 39 components were considered the RSNs of interest. Figure 1 shows the spatial content of included RSNs.

\section{Dynamic Functional Connectivity}

In addition to spatial information, a set of time courses characterize the temporal evolution of each RSN. The dFNC data were obtained using the sliding-time-windowcorrelation applied to RSN time courses (Louie and Wilson, 2001). Temporal window size was $40 \mathrm{~s}$ aiming at a fine resolution of temporal dynamics. Windowed correlations totaled 741 for each sliding TR shift. The data were 
Table I Subject Group Demographic Summary

\begin{tabular}{|c|c|c|c|c|c|c|c|c|c|}
\hline & CTR & SMK & DRN & SAD & MAR & SAM & MAD & ALL & Total \\
\hline Size & 51 & 80 & 250 & 47 & 13 & 17 & 51 & 25 & 534 \\
\hline Female \% & 35.3 & 46.3 & 39.6 & 25.5 & 23.1 & 29.4 & 33.3 & 16.0 & 36.50 \\
\hline \multicolumn{10}{|l|}{ Age } \\
\hline Min & 18 & 19 & 19 & 21 & 18 & 19 & 24 & 21 & 18 \\
\hline Mean & 34.5 & 34.8 & 33.4 & 34.0 & 28.8 & 29.8 & 31.9 & 29.2 & 33.2 \\
\hline Max & 54 & 54 & 55 & 53 & 49 & 41 & 55 & 52 & 55 \\
\hline AUDIT & $N / A$ & 0 & 8 & 8 & 0 & 0 & 8 & 8 & 0 \\
\hline \multicolumn{10}{|l|}{ Min } \\
\hline Mean & $N / A$ & 2.8 & 17.8 & 15.3 & 4.5 & 3.1 & 17.2 & 15.7 & 11.0 \\
\hline Max & $N / A$ & 7 & 39 & 32 & 7 & 6 & 33 & 32 & 39 \\
\hline FTQ & 0.0 & 7.0 & 0.0 & 7.0 & 0.0 & 7.0 & 0.0 & 7.0 & 0.0 \\
\hline \multicolumn{10}{|l|}{ Min } \\
\hline Mean & 0.0 & 9.0 & 0.6 & 9.0 & 0.8 & 9.6 & 0.6 & 8.2 & 4.7 \\
\hline Max & 0.0 & 13.0 & 6.0 & 12.0 & 5.0 & 13.0 & 4.0 & 11.0 & 13.0 \\
\hline Marijuana & 0.0 & 0.0 & 0.0 & 0.0 & 15.0 & 30.0 & 15.0 & 15.0 & 0.0 \\
\hline \multicolumn{10}{|l|}{ Min } \\
\hline Mean & 0.0 & 0.2 & 0.3 & 0.5 & 34.2 & 54.0 & 36.5 & 42.2 & 21.0 \\
\hline Max & 0.0 & 3.0 & 5.0 & 5.0 & 60.0 & 60.0 & 84.0 & 90.0 & 90.0 \\
\hline$B D /$ & $N / A$ & 0.0 & 0.0 & 0.0 & 0.0 & 0.0 & 0.0 & 0.0 & 0.0 \\
\hline \multicolumn{10}{|l|}{ Min } \\
\hline Mean & N/A & 5.3 & 7.9 & 8.4 & 4.8 & 6.9 & 7.7 & 10.6 & 7.4 \\
\hline Max & $\mathrm{N} / \mathrm{A}$ & 25.0 & 41.0 & 27.0 & 11.0 & 20.0 & 22.0 & 32.0 & 41.0 \\
\hline$B A I$ & $N / A$ & 0.0 & 0.0 & 0.0 & 0.0 & 0.0 & 0.0 & 0.0 & 0.0 \\
\hline \multicolumn{10}{|l|}{ Min } \\
\hline Mean & $\mathrm{N} / \mathrm{A}$ & 4.8 & 8.1 & 7.3 & 7.0 & 5.7 & 6.6 & 9.2 & 7.0 \\
\hline Max & $\mathrm{N} / \mathrm{A}$ & 24.0 & 42.0 & 53.0 & 23.0 & 23.0 & 26.0 & 28.0 & 53.0 \\
\hline ImpSS & $N / A$ & 1.0 & 0.0 & 1.0 & 3.0 & 1.0 & 1.0 & 2.0 & 0.0 \\
\hline \multicolumn{10}{|l|}{ Min } \\
\hline Mean & $N / A$ & 7.7 & 9.3 & 10.8 & 8.8 & 9.7 & 9.8 & 9.2 & 9.3 \\
\hline Max & $\mathrm{N} / \mathrm{A}$ & 18.0 & 19.0 & 19.0 & 17.0 & 18.0 & 19.0 & 19.0 & 19.0 \\
\hline Income & N/A & 1.0 & 1.0 & 1.0 & 1.0 & 1.0 & 1.0 & 1.0 & 1.0 \\
\hline \multicolumn{10}{|l|}{ Min } \\
\hline Mean & $\mathrm{N} / \mathrm{A}$ & 2.9 & 2.9 & 2.8 & 2.6 & 2.6 & 2.4 & 2.0 & 2.6 \\
\hline Max & $\mathrm{N} / \mathrm{A}$ & 7.0 & 7.0 & 7.0 & 7.0 & 6.0 & 7.0 & 7.0 & 7.0 \\
\hline \multicolumn{10}{|c|}{$\begin{array}{l}\text { Mean } \\
\text { Framewise displacement }\end{array}$} \\
\hline $\begin{array}{l}\text { ALL }>C T R \\
A L L>D R N\end{array}$ & 1.29 & 1.70 & 1.57 & 1.76 & 1.96 & 1.46 & 1.52 & 2.2 & 13.46 \\
\hline
\end{tabular}

structured such that one complete set of 741 windowed correlations represent the information of a single point in time. For simplicity, each set of 741 correlations in each TR shift will be named a window. Data from all subjects were clustered using the $k$-means clustering algorithm (Lloyd,
1982) to estimate the set of dFNC states in the data. The number of clusters was estimated to be 6 using the GIFT software package (Rachakonda et al, 2007). Figure 2 displays centroids for all six states where the difference in connectivity patterns is evident. 

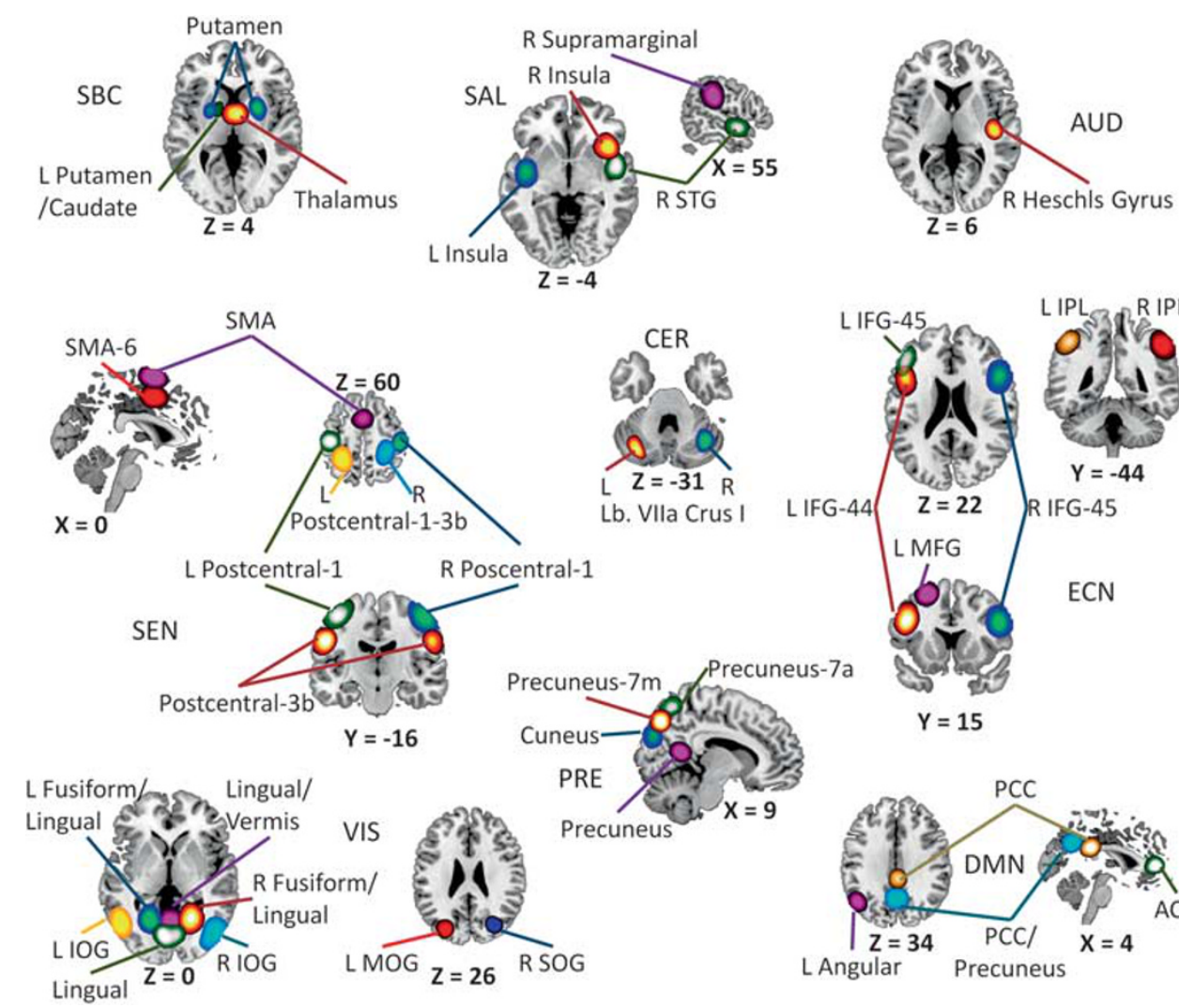

R Poscentral-1

b. VIlla Crus I
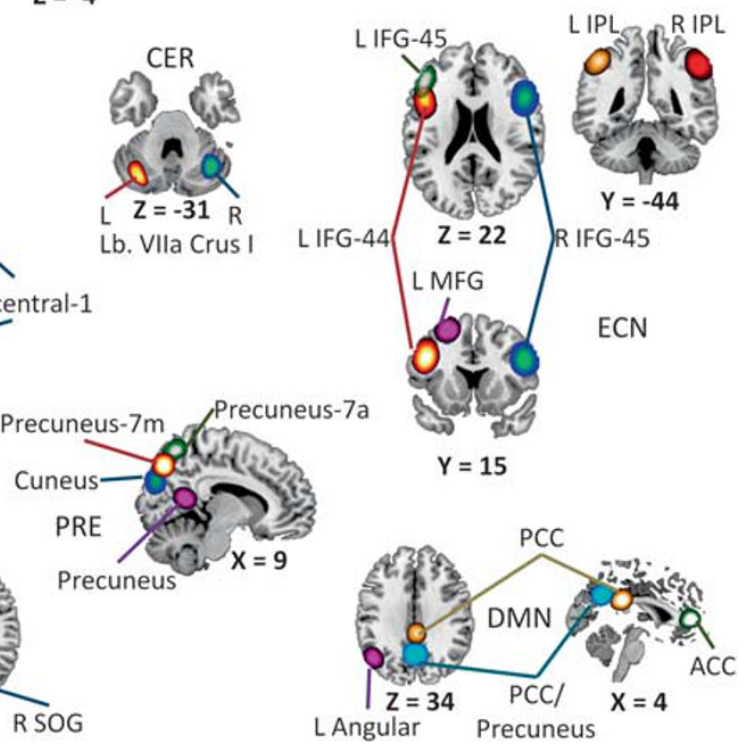

Figure I Spatial content of the resting state networks (RSNs) obtained from group independent component analysis (gICA). The gICA is able to decompose the fMRI data into several RSNs. Each RSN is a signal source with separate spatial and temporal information. The spatial part of the RSN delimits a brain region of interest in the study. The different colors in the picture aid differentiating RSNs from one another. It is common in gICA to have some RSNs composed of more than one brain area. In some cases, one RSN includes left and right content of the same brain area. RSNs were grouped according to their functional relevance in subcortical (SBC), cerebellum (CER), auditory (AUD), sensorimotor (SEN), visual (VIS), salience (SAL), default mode network (DMN), executive control network (ECN), and precuneus (PRE). MNI coordinates for the peaks of all RSNs and corresponding groups are available as Supplementary Material.

\section{RESULTS}

\section{Substances Affecting the Occupancy Rates of dFNC States}

Subject-wise occupancy rates were determined by the frequency with which each $\mathrm{dFNC}$ state appeared on each subject. Occupancy rate differences for each of the eight substance groups were then tested utilizing six one-way ANOVAs. The ANOVA tests for state $2 \quad(\mathrm{~F}=2.49$, $P=0.0161)$ and state $4(\mathrm{~F}=2.47, P=0.0170)$ were significant. ANOVA tables are provided in Supplementary Material. Figure 3 displays mean occupancy rates and post hoc results from Fisher's least significant difference (LSD) (Fisher, 1937). After detecting a significant ANOVA, LSD finds the smallest significant difference and considers larger differences as significant (Williams and Abdi, 2010). The DRN group showed larger occupancy than CTR and ALL groups in state 4. The MAR group also showed larger occupancy than the ALL group. In contrast, DRN and MAD groups had lower occupancy than the CTR group in state 2. The SAM group had larger occupancy rate than DRN. In both states, MAR and DRN groups follow the similar tendency with high occupancy in state 2 and low occupancy in state 3 , but because of its large variance the MAR group had no significant differences with other groups in state 2 .
A linear regression model was applied with the occupancy rate as dependent variable (DV). Results from linear regression models displayed in Table 2 show a significant and negative slope between occupancy rates and AUDIT in state 2, agreeing with the ANOVA post hoc analysis in Figure 3. However, state 4 had no significant regression coefficients of substance-related scores. There were no significant coefficients for depression (BDI), anxiety (BAI), and sensation seeking (ImpSS) on any of the six states. State 2 had significant relationships with sex and income, but AUDIT had the larger effect size. The largest covariate in state 4 was age with a very strong effect size, followed by income as the second strongest covariate. Movement noise on the $z$-axis was significant in state 4 and might have contributed to the lack of effects related to substances.

The strongest result indicates that drinkers and comorbid alcohol-cannabis users exhibit a reduced influence in state 2 , where connectivity among saliency, motor, and sensorial processing is strong. Connectivity for the cannabis group follows a similar trend, but the lack of significance may be influenced by the differences in sample sizes. Nicotine and comorbid nicotine-cannabis did not show an effect or a trend in this occupancy rate analysis. 

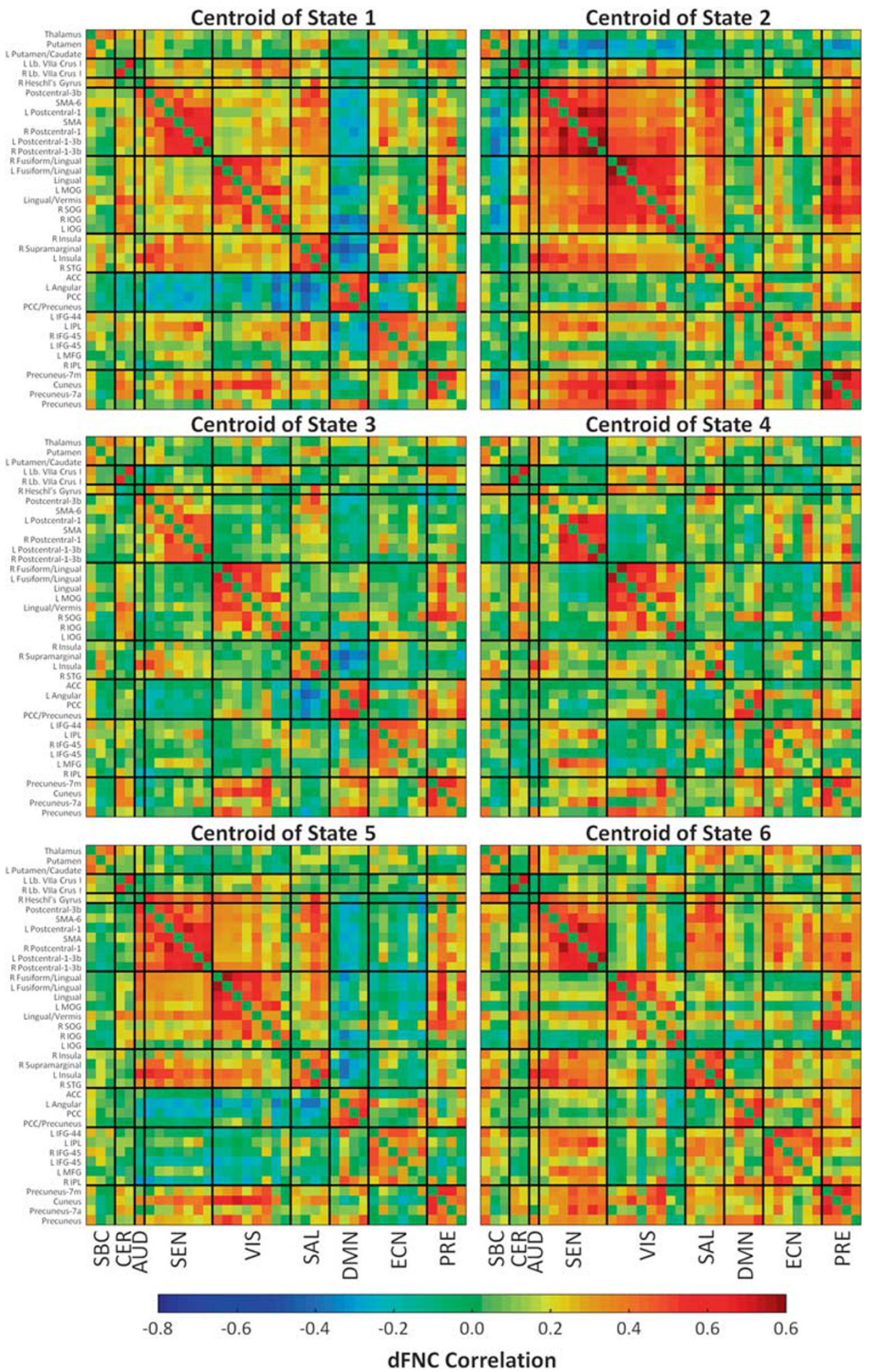

Figure 2 Dynamic functional network connectivity ( $\mathrm{dFNC}$ ) assumes that whole brain connectivity sequentially iterates through a finite set of connectivity patterns also known as dFNC states. In practice, the states might present some differences according to the subject and the particular moment in time. The picture displays mean connectivity matrices, called centroids, obtained from all subjects and moments in time with the same state. Anticorrelation between DMN and other RSNs characterizes state I. Centroid 2 features high correlation among networks recruited outside resting state. Centroids 3 and 4 are similar and depict high correlation within most RSN groups, but low correlation between RSN groups. Centroid 3 includes a particular anticorrelation between salience (SAL) and DMN. Centroid 5 is similar to centroid I except for a low correlation pattern of executive control RSNs. Finally, centroid 6 presents a particular pattern of positive correlations that is not observed in the previous centroids. 


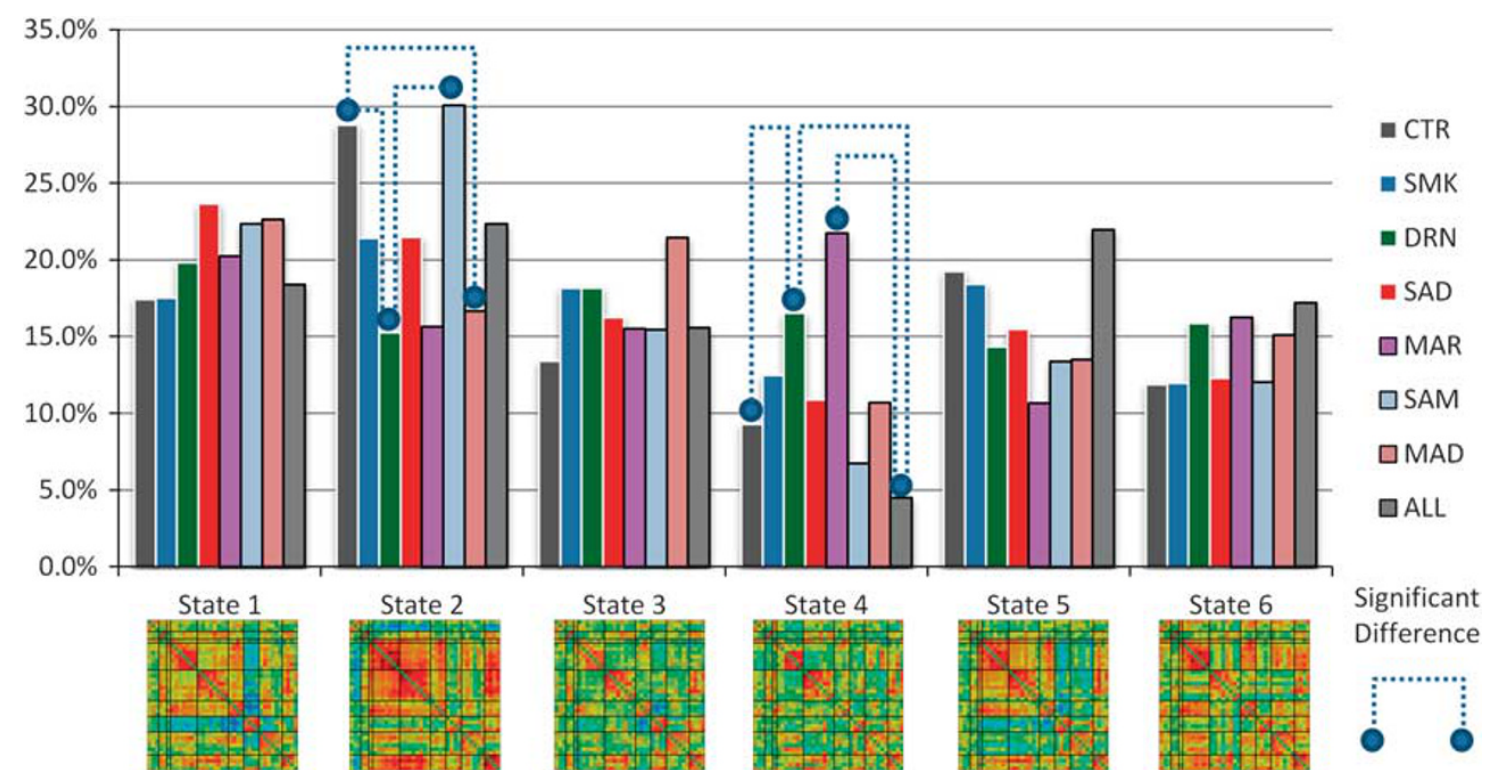

Figure 3 The post hoc analysis for cluster occupancy rates on each state. A dFNC analysis assumes different connectivity patterns that sequentially change through time. The ratio of the number of times a state appears in a subject's brain over the total scanning time is known as the occupancy rate. Some subjects might have higher occupancy rates in one state indicating their preference for that connectivity pattern. The figure displays differences in state preference, assessed as occupancy rates, based on substance use groups. Bars display the means of each substance group. The dotted line indicates significant difference based on ANOVA tests and least significant different post hoc.

\section{Substance Effects in dFNC Strength}

A multivariate analysis of variance (MANOVA) (Warne, 2014) was used in each state to determine group differences using correlations from all $741(39 \times 38 / 2)$ RSN pairs as DVs. Windows belonging to the same dFNC state were averaged for each subject to represent the subject on a particular state. Within a dFNC state, the 741 average values of each subject entered MANOVA as DVs. MANOVA results were all significant $(P<1$ e-94) indicating at least one group difference. Specifics about the MANOVA results are included in the Supplementary Material. The post hoc analyses were performed using linear discriminant analysis (LDA). LDA is a multivariate method, a generalization of Fisher's linear discriminant (Iatan, 2010), used to find linear combinations of DVs that best separate the groups. In our context, LDA delivers a finite set of vectors (termed canonical vectors) with subject corresponding values. For ease of illustration, we only utilized the first two canonical vectors for the scatter plots displayed in Figure 4 to show group separation in a twodimensional space. The scatter plot also includes a vector indicating the direction of increasing connectivity strength. Connectivity strength is a global measure of connectivity obtained by averaging all available correlations (Lynall et al, 2010). The global characteristic of connectivity strength is appropriate for this analysis given that LDA is a linear combination of connectivity values spanning the whole brain. The connectivity strength vector was obtained by averaging the $741 \mathrm{dFNCs}$ on each state and calculating the projection to the corresponding canonical vectors. In this analysis, only the vector direction is important and points to increasing dFNC connectivity strength. Two dimension scatter plots illustrate subject group segregation where all eight groups are almost perfectly separated. However, this visualization does not show dFNC information of all seven dimensions. Figure $4 \mathrm{~h}$ exhibits the distribution of subject groups along the projection of the connectivity strength vector in seven dimensions. The way this projection was calculated is exemplified in Figure 4g. Figure $4 \mathrm{~h}$ allows for the visualization of the relationship between subject group and connectivity strength. The results illustrate the possibility of discriminating between the eight groups by means of a multivariate analysis and performing specific linear combinations of dFNC values. In state 1, the MAR and ALL groups show larger connectivity strength than the rest, but the SAM group exhibits a lower value. In states 2, 3, and 5, the MAR group has noticeably larger connectivity strength than the others. In state 2, CTR and SAM groups have the second strongest connectivity strength. Besides the MAR group, the other subject groups do not differentiate along the connectivity strength axis. State 4 exhibits a SAM group with larger connectivity strength than the other groups. State 5 has the most confounded subject groups of all states. State 6 shows low connectivity strength in the SAM and DRN groups; the CTR group has higher connectivity strength than the other groups.

Because canonical vectors are linear combinations of the dFNCs, it is difficult to see changes in connectivity for single RSN pairs. Specific connectivity effects were analyzed using ANOVAs on AFNC values (one ANOVA for each one of the 741 dFNCs) seeking significant group differences $(P<0.05)$ and correcting using false discovery rate (FDR). Finally, a linear regression model was applied to the subset of 741 connectivity values with significant ANOVA. The linear model assumed the connectivity between a particular RSN pair as DV. The significant ANOVA results are displayed in Figure 5 and regression model results are presented in Table 3. Movement covariates based on the mean frame-wise displacement of each coordinate were included to correct 
and diminish possible movement variance in the analysis. Only the result from state 1 had a significant noise related to the $y$-axis movement, but the inclusion of this covariate reduced the influence of movement. The other results in Table 3 did not exhibit a significant movement variance. Group results for states 1 and 2 were consistent with a reduction of dFNC because of alcohol. In both states, the DRN group presented a lower dFNC mean with respect to CTR and SMK groups. At the same time, both states present a significant negative slope with AUDIT increasing the evidence that alcohol use is related to this diminished connectivity. The result from state 1 feature connectivity between the left inferior frontal gyrus (L. IFG) and the right postcentral gyrus. The particularity of this result is a significant relationship with cannabis consumption. It might explain in part the increased mean dFNC in the ALL group and the difference between DRN and MAD groups. The result from state 2 include the connectivity between a RSN comprising right fusiform and lingual giri, and a RSN with supplementary motor area (SMA) content. The relationship between CTR, DRN, and SMK groups is the same as in state 1 . The difference can be seen in a lack of a significant marijuana link and the ALL group exhibits a decreased dFNC. For the ALL group, alcohol use seems to lead the direction of the effect in state 2 as opposed to the strong influence of marijuana use seen in state 1.

Four ANOVAs were significant in state 4 involving the connectivity between dorsal striatum and sensorimotor regions. Groups of nicotine use (SMK, SAD, SAM, and ALL) had no significant difference in dFNC for the pair putamen and SMA. With some few exceptions, these four nicotinic groups exhibited a lower dFNC than the nonnicotine groups including CTR. The regression analysis reveals a strong effect (22.9\% of variance explained) of reduced connectivity with increased FTQ. These results suggest a strong connectivity reduction between putamen and SMA linked to nicotine use. An almost identical pattern can be observed for the RSN pair L.Putamen/Caudate and L. Postcentral-1-3b where the effect size for FTQ is also significant (10\% of variance explained). Although similar in trend and anatomical regions, the other two RSN pairs show a significant link with nicotine use. However, these results exhibited a lack of group difference between CTR and SMK groups. Except for a significant link to AUDIT in the pair L.Putamen/Caudate $v s$ L.Postcentral-1, no other covariate had a significant effect on dFNC. The dFNC in state 4 seems to be mainly influenced by nicotine use.

\section{DISCUSSION}

Drawing from the poly-substance characteristics of the subjects considered in this study, one important objective of this work was to detect dFNC differences linked to combinations of the three commonly used substances. In general, the data were rich enough to allow for a match of substance use and dFNC characteristics acquired through appropriate linear combinations. These linear combinations do not point to a particular gray matter region, but represent the aggregated contribution of brain areas spanning the whole brain. Figure $4 a-f$ illustrates 2 out of the 7 available data dimensions with significant information about 
a

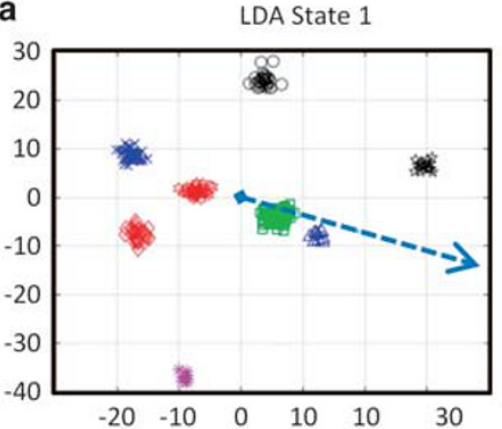

d

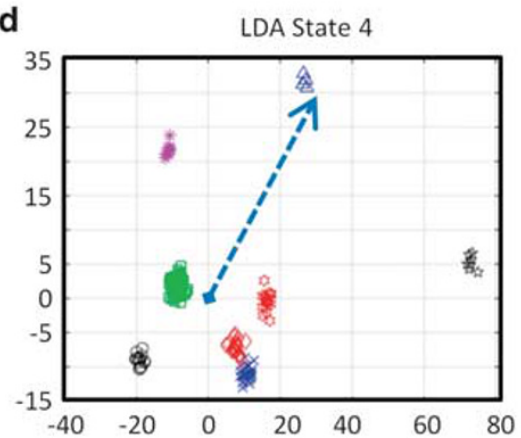

b

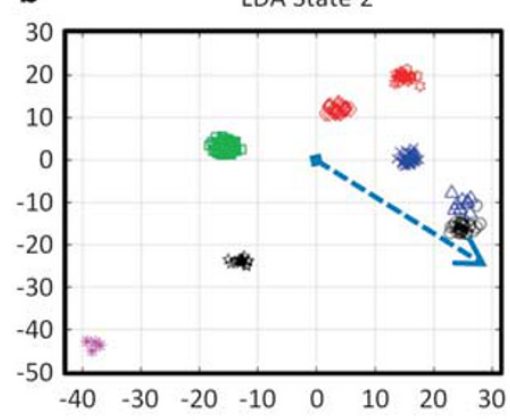

e

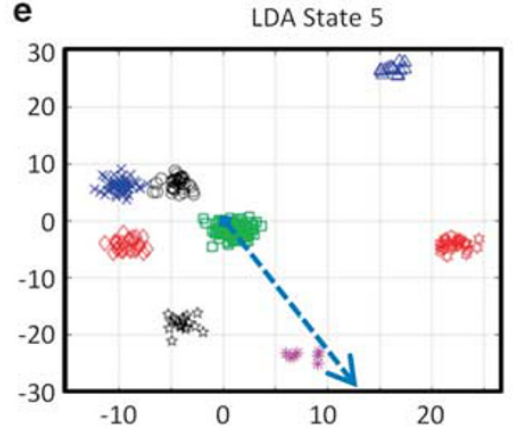

C

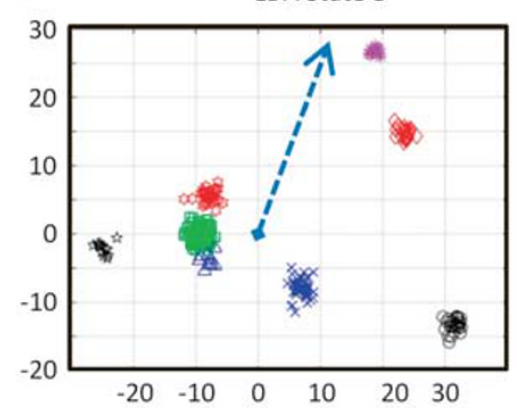

f

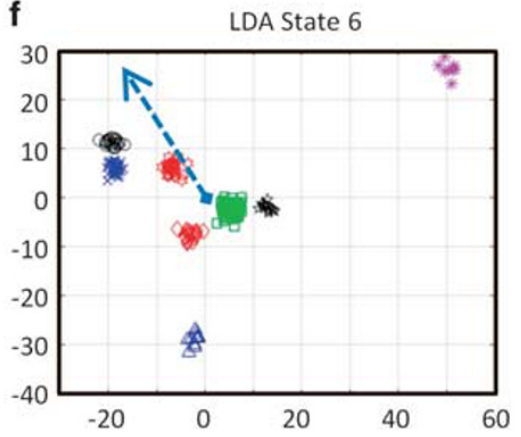

g

h

Projection along Global Connectivity Strength

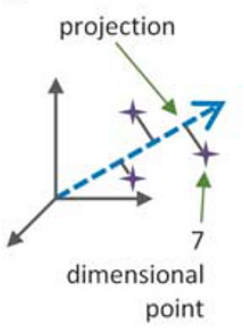

Calculate Projection

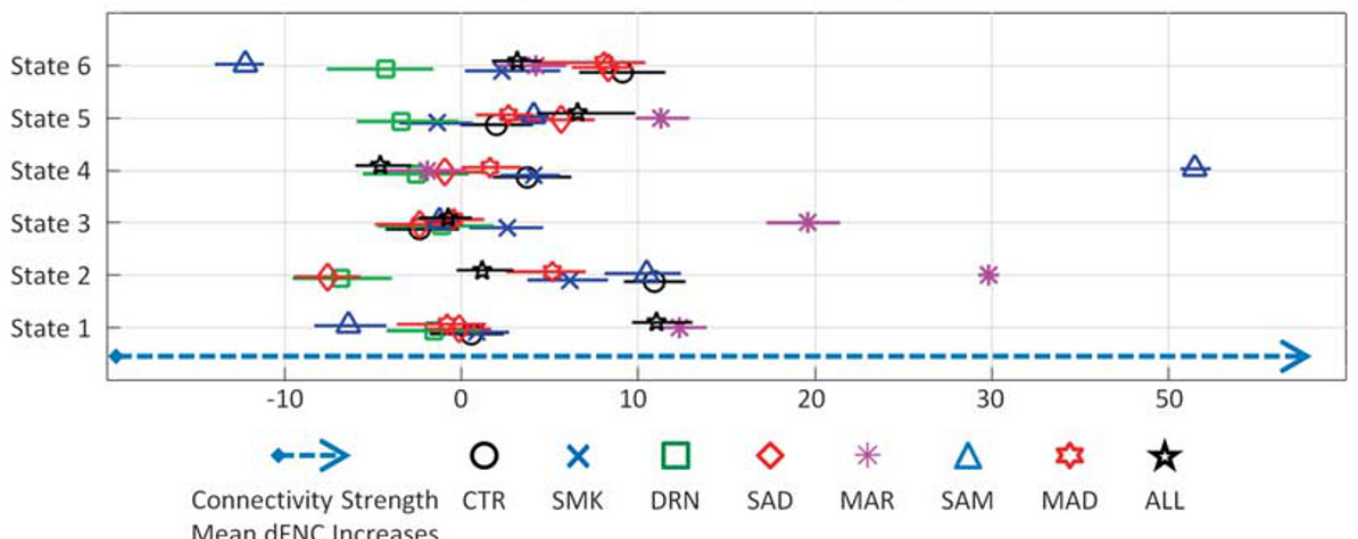

Figure 4 The post hoc analyses of MANOVAs performed on all states indicate the existence of significant group differences. The post hoc analysis in this figure corresponds to a linear discriminant analysis (LDA). LDA finds linear combinations of connectivity across the whole brain that best separate the sample groups. Figure $4 \mathrm{a}-\mathrm{f}$ utilizes the first two canonical vectors, outcomes of LDA linear combinations, to illustrate the ability to separate sample groups. Axes $x$ and y represent the first and second canonical vectors respectively. To better understand the data, we calculated the representative connectivity strength vector (indicating the direction of global increment of connectivity, ie, connectivity increment of the whole brain) on each state for the first seven canonical vectors. The coordinates of each subject were then projected over the connectivity strength vector on each state. Figure $4 \mathrm{~g}$ depicts the concept of coordinate projection onto the connectivity strength vector. ANOVAs on the projection value as response were significant for all state $\left(P<1.2 \times 10^{-31}\right)$ confirming observed group differences. Figure $4 \mathrm{~h}$ displays a summary of substance group projections. The marker is positioned at the mean value. The line crossing the marker ranges from the minimum to the maximum projected coordinate.

differences in the $741 \mathrm{dFNC}$ measures. These scatter plots show that each substance or their combined use has separate and identifiable effects in the brain. The difficulty in interpreting MANOVA results comes from its multivariate and higher dimensional features. As an outcome of the aggregation of connectivity through the whole brain, it is reasonable to compare the results with a global measure of connectivity. Figure $4 \mathrm{~h}$ presents an interpretable visualization of the results based on global connectivity strength measure. Connectivity differences could be seen at the global level that we discuss in the following paragraphs along with more localized effects.

The overall effect of alcohol is a reduction of dFNC, especially among motor and sensorial areas. Figure 3 shows evidence that alcohol drinkers avert state 2 , where there is a strong connectivity among sensorimotor, salience, and precuneus brain areas. The lack of preference for state 2 is confirmed by the negative regression coefficient that was found significant for the occupancy rate of state 2 in Table 2. The connectivity pattern in state 2 is in line with the hypothesis of an extrospective mind state within the resting 


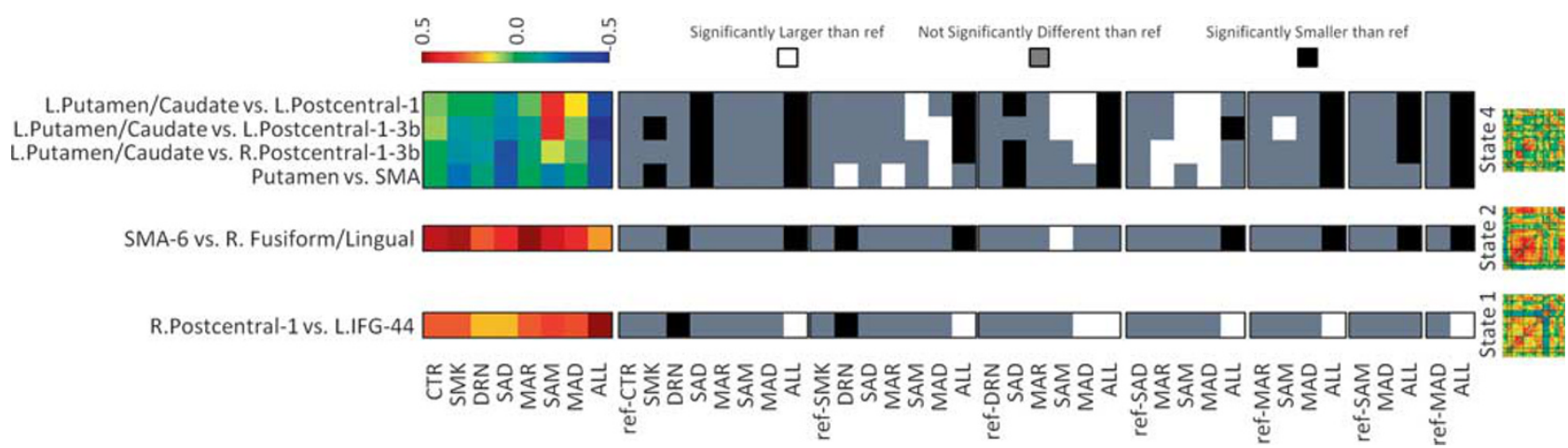

Figure 5 Histogram summarizing significant ANOVAs. An ANOVA was performed for each of the 74I available correlations. Only six group differences were found after false discovery rate correction on ANOVAs P-values. The Fisher $r$-to-z transformation was applied to all correlation values and the color scale was restricted to the range [ -0.5 to 0.5]. Pairwise group comparisons are indicated in black and white. The reference group on each block in marked as 'ref.' Lower connectivity in the alcohol DRN group compared with controls (CTR) was found in states I and 2. Lower connectivity in nicotine smokers (SMK), smoke-and-drink (SAD), and all substances (ALL) groups compared with the CTR group characterized state 4.

state, providing readiness in case attention to outside stimuli is necessary (Fransson, 2005). In contrast, there is an inflated preference for state 4 , where the mentioned brain areas are weakly connected. Not only the dFNC state preference is different for drinkers, but also connectivity strength. Figure $4 \mathrm{~h}$ presents additional connectivity reduction in most states including states 2 and 4 . In addition to whole brain connectivity data, univariate analyses identified reduced dFNC in drinkers between visual and motor areas, agreeing with the multivariate outcomes. Linear model results in this study provide previously missing evidence (Vergara et al, 2017a) of a significant link between alcohol effects in functional connectivity with alcohol use measures; specifically, the AUDIT score. This set of consistent results observed in dFNC also agrees with former studies of static functional connectivity. A previous study found a reduction of static functional connectivity primarily among the insula, precuneus, sensorimotor, and visual areas, but an increase on the putamen after testing for group differences against nondrinkers (Vergara et al, 2017a). These outcomes support both the 'disconnection syndrome' (Dupuy and Chanraud, 2016) and the reduced interoception effect (Çöl et al, 2016) related to alcohol use. Alcohol-related functional disconnection has been reported by several studies (Weiland et al, 2014) as associated with drinking, abstinence, and relapse (Camchong et al, 2013a, b) and loss of network efficiency in the brain (Sjoerds et al, 2017). This and the previously mentioned studies only presented evidence of functional disconnection, but structural studies have also indicated decreased white matter integrity (Jansen et al, 2015; Kril et al, 1997; Yeh et al, 2009); this helps explain the overall extent of the disconnection syndrome. One important point is that observed functional disconnection is more prominent in state 2, where salient brain areas in the insula (see the insula in Figure 1 and state 2 in Figure 2) are strongly connected with other brain regions. The insula is actively involved in interoception because it is a structure that processes the physiological condition of the rest of the body (Craig, 2003). The results are in accordance with the idea of a reduced interoception because of the disconnection produced by alcohol use. Evidence of reduced iteroception awareness related to alcohol use has been previously presented (Çöl et al, 2016) by means of a heartbeat perception performance method. In addition to alcohol, our data suggest that marijuana consumption may produce aberrant interoception patterns because of an increased occupancy rate in state 4 (see Figure 3) where the insula is weakly connected to the rest of the brain. Connectivity strength was also reduced in marijuana subjects within state 4 (see Figure 4); however, state 2 presents the opposite effect with different outcome than alcohol drinkers. It has been theorized that aberrant interoception is an effect that belongs to all addiction in general (Verdejo-Garcia et al, 2012). In this respect, our data suggest that alcohol may produce one of the largest aberrant interoception among the substances of abuse. We can turn our attention to state 1 , where univariate analysis found a decrease in dFNC between a frontal area of the ECN and postcentral gyrus; both task-positive networks (TPNs). TPNs are brain networks elicited to perform demanding tasks (Fox et al, 2005). Aberrant connectivity in the ECN has been suggested as a contributing factor in sustaining alcohol addiction (Weiland et al, 2014). Group differences found in our analyses agree in part with predictions of the network model of addiction where resting state connectivity among TPNs and between salience and TPNs are reduced after substance use (Sutherland et al, 2012). In summary, alcohol use produces a general resting state functional disconnection that is harsh on TPNs (including sensorimotor and executive control areas) and the insula, a region important for interoceptive functions.

Concurrent nicotine and alcohol consumption did not have an effect in occupancy rates. The SAD group exhibited similar connectivity strength as the DRN group in states 2 and 4. There was no similar trend between SMK and SAD groups. In states 1 and 3, the two groups had connectivity strength similar to controls indicating the absence of an important effect. The similarity of effect between populations that drink and those that concurrently smoke and drink has been observed before in studying static FNC (Vergara et al, 2017a). In that static FNC study, there was a sparing of high visual areas in SAD subjects. A similar outcome was observed in Figure 5, where the connectivity between motor and a high visual processing area was reduced in drinkers, but was unaffected in SAD subjects. This outcome could be 
Table 3 Results from Linear Regression Models for dFNC on Each State

\begin{tabular}{|c|c|c|c|c|c|c|c|c|c|c|c|c|c|c|c|c|c|c|}
\hline State & & I & & & 2 & & & 4 & & & 4 & & & 4 & & & 4 & \\
\hline \multirow{3}{*}{$\begin{array}{l}\text { RSN Pair } \\
\text { RSN No. }\end{array}$} & \multicolumn{3}{|c|}{ L.IFG-44 } & \multicolumn{3}{|c|}{ R.Fusiform/Lingual } & \multicolumn{3}{|c|}{ SMA } & \multicolumn{3}{|c|}{ R.Postcentral-I-3b } & \multicolumn{3}{|c|}{ L.Postcentral-I-3b } & \multicolumn{3}{|c|}{ L.Postcentral-I } \\
\hline & \multicolumn{3}{|c|}{ R.Postcentral-I } & \multicolumn{3}{|c|}{ SMA-6 } & \multicolumn{3}{|c|}{ Putamen } & \multicolumn{3}{|c|}{ L.Putamen/Caudate } & \multicolumn{3}{|c|}{ L.Putamen/Caudate } & \multicolumn{3}{|c|}{ L.Putamen/Caudate } \\
\hline & $\beta$ & $P$ & $\%$ Var & $\beta$ & $P$ & $\%$ Var & $\beta$ & $P$ & $\%$ Var & $\beta$ & $P$ & $\%$ Var & $\beta$ & $P$ & $\%$ Var & $\beta$ & $P$ & $\%$ Var \\
\hline Constant & $3.4 \mathrm{E}-01$ & 0.01 & $0.0 \%$ & $6.5 \mathrm{E}-0 \mathrm{I}$ & $2.4 \mathrm{E}-06$ & $0.0 \%$ & $4.5 \mathrm{E}-02$ & 0.75 & $0.0 \%$ & $-2.4 \mathrm{E}-0 \mathrm{I}$ & 0.14 & $0.0 \%$ & $-1.9 \mathrm{E}-01$ & 0.32 & $0.0 \%$ & $8.1 E-02$ & 0.63 & $0.0 \%$ \\
\hline Sex & $-4.3 E-02$ & 0.40 & $0.4 \%$ & $3.3 E-02$ & 0.54 & $0.3 \%$ & $4.0 \mathrm{E}-02$ & 0.44 & $0.5 \%$ & $1.6 \mathrm{E}-02$ & 0.79 & $0.1 \%$ & $4.5 E-02$ & 0.52 & $0.4 \%$ & $6.8 \mathrm{E}-03$ & 0.91 & $0.0 \%$ \\
\hline Age & $-4.7 \mathrm{E}-03$ & 0.11 & $1.3 \%$ & $-5.1 E-03$ & 0.08 & $2.2 \%$ & $1.3 \mathrm{E}-03$ & 0.67 & $0.2 \%$ & $5.0 \mathrm{E}-03$ & 0.16 & $2.3 \%$ & $5.9 \mathrm{E}-03$ & 0.15 & $2.7 \%$ & $1.4 \mathrm{E}-03$ & 0.70 & $0.2 \%$ \\
\hline $\begin{array}{l}\text { Marijuana } \\
\text { 60TLFB }\end{array}$ & $2.5 \mathrm{E}-03$ & $3.6 \mathrm{E}-02$ & $2.0 \%$ & $2.4 \mathrm{E}-04$ & 0.86 & $0.0 \%$ & $5.3 E-04$ & 0.73 & $0.1 \%$ & $3.5 E-03$ & 0.05 & $3.6 \%$ & $2.5 E-03$ & 0.22 & $1.5 \%$ & $2.4 \mathrm{E}-03$ & 0.19 & $1.5 \%$ \\
\hline FTQ & $-7.8 \mathrm{E}-04$ & 0.89 & $0.0 \%$ & $1.2 \mathrm{E}-02$ & 0.06 & $3.1 \%$ & $-2.9 E-02$ & $3.0 \mathrm{E}-05$ & $22.9 \%$ & $-2.0 \mathrm{E}-02$ & I.IE-02 & $9.3 \%$ & $-2.4 \mathrm{E}-02$ & $9.5 E-03$ & $10.1 \%$ & $-2.5 E-02$ & $2.3 \mathrm{E}-03$ & $13.1 \%$ \\
\hline AUDIT & $-8.1 E-03$ & 4.7E-02 & $2.8 \%$ & $-7.9 E-03$ & $4.3 E-02$ & $3.9 \%$ & $-2.2 E-03$ & 0.58 & $0.4 \%$ & $-5.7 E-03$ & 0.21 & $2.3 \%$ & $-5.2 E-03$ & 0.31 & $1.6 \%$ & $-9.8 E-03$ & $3.5 E-02$ & $6.2 \%$ \\
\hline $\mathrm{BDI}$ & $1.9 \mathrm{E}-03$ & 0.68 & $0.1 \%$ & $-4.8 \mathrm{E}-03$ & 0.20 & $1.2 \%$ & $6.6 \mathrm{E}-04$ & 0.86 & $0.0 \%$ & $4.3 \mathrm{E}-03$ & 0.34 & $1.1 \%$ & $-4.8 \mathrm{E}-04$ & 0.92 & $0.0 \%$ & $6.5 \mathrm{E}-04$ & 0.89 & $0.0 \%$ \\
\hline ImpSS & $2.0 \mathrm{E}-03$ & 0.69 & $0.1 \%$ & $-8.3 \mathrm{E}-03$ & 0.14 & $1.6 \%$ & $-1.3 E-03$ & 0.82 & $0.1 \%$ & $-5.5 E-04$ & 0.93 & $0.0 \%$ & $2.9 \mathrm{E}-03$ & 0.70 & $0.2 \%$ & $4.4 \mathrm{E}-03$ & 0.51 & $0.5 \%$ \\
\hline BAl & $2.4 \mathrm{E}-03$ & 0.59 & $0.2 \%$ & ।.7E-04 & 0.56 & $0.2 \%$ & $3.2 \mathrm{E}-04$ & 0.23 & $1.2 \%$ & $1.9 \mathrm{E}-04$ & 0.52 & $0.4 \%$ & $2.3 E-04$ & 0.50 & $0.4 \%$ & $-4.2 \mathrm{E}-05$ & 0.89 & $0.0 \%$ \\
\hline Income & $1.3 \mathrm{E}-02$ & 0.33 & $0.4 \%$ & $3.2 \mathrm{E}-02$ & $2.0 \mathrm{E}-02$ & $3.5 \%$ & $1.2 \mathrm{E}-02$ & 0.44 & $0.5 \%$ & $-1.1 E-03$ & 0.95 & $0.0 \%$ & $1.4 \mathrm{E}-02$ & 0.50 & $0.4 \%$ & $6.6 \mathrm{E}-03$ & 0.73 & $0.1 \%$ \\
\hline x & $7.7 E-04$ & 0.59 & $0.3 \%$ & $-6.7 \mathrm{E}-05$ & 0.96 & $0.0 \%$ & $7.8 \mathrm{E}-04$ & 0.59 & $0.8 \%$ & $2.5 E-04$ & 0.88 & $0.1 \%$ & $8.5 E-04$ & 0.66 & $0.7 \%$ & $1.9 \mathrm{E}-03$ & 0.26 & $3.9 \%$ \\
\hline y & $4.0 E-03$ & 1.7E-03 & $7.9 \%$ & $-1.2 E-03$ & 0.31 & $1.3 \%$ & $-1.2 \mathrm{E}-03$ & 0.32 & $1.6 \%$ & $-2.6 \mathrm{E}-03$ & 0.05 & $6.6 \%$ & $-2.3 E-03$ & 0.15 & $3.9 \%$ & $-2.2 E-03$ & 0.11 & $4.4 \%$ \\
\hline$z$ & $-3.9 E-04$ & 0.61 & $0.2 \%$ & $-7.9 E-07$ & 1.00 & $0.0 \%$ & $-9.0 E-04$ & 0.17 & $2.8 \%$ & $-1.8 \mathrm{E}-04$ & 0.81 & $0.1 \%$ & $-7.6 \mathrm{E}-04$ & 0.38 & $1.4 \%$ & $-1.2 \mathrm{E}-03$ & 0.12 & $3.9 \%$ \\
\hline$x$-rot & $2.4 \mathrm{E}-04$ & 0.66 & $0.1 \%$ & $-1.6 \mathrm{E}-04$ & 0.73 & $0.1 \%$ & $8.0 \mathrm{E}-05$ & 0.88 & $0.0 \%$ & $1.2 \mathrm{E}-03$ & 0.05 & $7.2 \%$ & I.IE-03 & 0.11 & $5.2 \%$ & $1.2 \mathrm{E}-03$ & 0.06 & $6.5 \%$ \\
\hline$y$-rot & $-9.5 E-04$ & 0.24 & $0.7 \%$ & $1.0 \mathrm{E}-03$ & 0.23 & $1.2 \%$ & $-2.1 E-06$ & 1.00 & $0.0 \%$ & $4.0 E-04$ & 0.62 & $0.4 \%$ & $-3.7 E-04$ & 0.68 & $0.2 \%$ & $4.5 E-04$ & 0.59 & $0.4 \%$ \\
\hline$z$-rot & $-4.8 \mathrm{E}-04$ & 0.68 & $0.2 \%$ & $-6.5 E-04$ & 0.57 & $0.4 \%$ & $-9.0 \mathrm{E}-04$ & 0.49 & $0.9 \%$ & $4.8 \mathrm{E}-04$ & 0.75 & $0.2 \%$ & $3.9 E-04$ & 0.82 & $0.1 \%$ & $1.5 E-04$ & 0.93 & $0.0 \%$ \\
\hline
\end{tabular}

Regression models were only assessed for FDR significant ANOVAs. Shaded regions indicate significant results $(P<0.05$ uncorrected) 
related to the activation enhancement of high visual areas produced by nicotine (Ghatan et al, 1998; Lawrence et al, 2002). The effect of nicotine on alcohol drinkers might have diminished dysfunctional connectivity because of alcohol, but future research is needed to verify the existence of this effect.

Nicotine outcomes were fewer overall, but with some relatively large effect sizes. Although no group differences in occupancy rate were found in relation to the SMK group, significant associations with FTQ were observed in the linear model. Higher occupancy rates in state 5 were linked to larger FTQ values. Lower occupancy rates in state 6 were associated with larger FTQ. The main difference between these two states is the higher connectivity of the ECN and the $\mathrm{DMN}$ in state 6 as compared with state 5 , suggesting that nicotine reduces connectivity of the ECN and the DMN. Connectivity strength results in Figure 4 show a tendency for connectivity reduction in states 2,5 , and 6 when comparing SMK and CTR groups, favoring a reduced connectivity because of nicotine use. However, connectivity strength has a contrasting picture of increased connectivity in state 3 and no difference with controls in states 1 and 4 . These contrasting results are not completely unexpected as nicotine has been found to produce both increased and decreased connectivity in some brain areas, including the frontoparietal network and the DMN (Pariyadath et al, 2014). Univariate analysis produced a more conclusive set of outcomes. ANOVA results in Figure 5 show a significant dFNC decrease in smokers compared with controls between sensorimotor and dorsal striatum areas in state 4 . Reduced $\mathrm{dFNC}$ in nicotine users is further verified by significant links between FTQ and striatal-sensorimotor connectivity displayed in Table 3 with large effect sizes characterized by percentages of variance explained between $13 \%$ and $22 \%$. To the best knowledge of the authors, this is one of the few times a strong effect of resting state functional connectivity has been observed in the dorsal striatum linked to nicotine. Static connectivity analysis using seed-based methods found that smokers during an abstinent period of $24 \mathrm{~h}$ exhibit decreased connectivity between dorsal striatum and cortical regions that include the supplementary motor area (Sweitzer et al, 2016). Although the ventral striatum is more frequently associated with nicotine addiction because of its role in the dopamine pathway (Brody et al, 2004; Okita et al, 2016), the dorsal striatum is thought to become a more important player as drug seeking transitions from voluntary to habitual behavior (Everitt and Robbins, 2005). This transition has been suggested to be present in abstinent nicotine smokers as an underlying mechanism that suppresses some automated habitual conduct in favor of diverting resources to craving and nicotine seeking behavior (Sweitzer et al, 2016; Tiffany and Conklin, 2000). There is also evidence that the dorsal, and not the ventral, striatum suffer morphological changes (volume and surface area) associated with nicotine craving (Janes et al, 2015). Our data and the previously mentioned studies support the existence of structural and functional connectivity changes in the dorsal striatum (putamen and caudate) linked to nicotine use and dependence.

The connectivity strength of marijuana subjects was larger than controls in states $1,2,3$, and 5 , but lower with a smaller magnitude in the other two states as displayed in Figure $4 \mathrm{~h}$. This result indicates that marijuana induces a stronger increment of connectivity through the brain, in selected dFNC states, than decrements. The effects were observed in a whole brain connectivity summary, but were not observable when selecting specific brain areas. Figure 5 illustrates this observation in the boxes comparing the CTR group with the rest. It can be argued that two factors contributed to the small number of marijuana results: (1) the large number of comparisons that were corrected and (2) the small number of marijuana subjects. Nevertheless, multivariate group results were observed as they are based on the linear combination of contributions from many group differences that were excluded if applying statistical multicomparison correction. Increased functional connectivity in cannabis users compared with controls has been previously reported in areas including the orbitofrontal cortex (Filbey et al, 2014); precentral, middle frontal, superior frontal, cingulate, inferior frontal, and fusiform giri (Cheng et al, 2014); and posterior cingulate and insula (Pujol et al, 2014). These observations of functional connectivity increments, including that in our data, are not expressions of beneficial effects. Structural studies found a series of axonal impairment in the hippocampus (fornix), splenium, commissural fibers (Zalesky et al, 2012), and morphological changes in the amygdala (Cousijn et al, 2012), cerebellum (Cousijn et al, 2012; Medina et al, 2010), and prefrontal cortex (Medina et al, 2009). One hypothesis that can explain why increased functional connectivity might point to an actual dysfunction suggests interference of some brain network in the normal function of others (Sutherland et al, 2012). This idea has been used to explain increased connectivity in key areas of the default mode network as a detrimental effect on the brain (Pujol et al, 2014).

As previously explained, both marijuana and alcohol consumptions are linked to changes of structural connectivity (Jansen et al, 2015; Zalesky et al, 2012), but affect functional connectivity in opposite directions as alcohol decreases (Camchong et al, 2013b; Vergara et al, 2017a; Weiland et al, 2014) and marijuana increases (Cheng et al, 2014; Filbey et al, 2014; Pujol et al, 2014) overall connectivity. Thinking in an additive way, the effect of concurrent use of these substances may subtract each other and this trend should be observable in the MAD sample group. Connectivity strength measures in Figure $4 \mathrm{~h}$ support this view, giving the trend of the MAD group to be closer to controls (CTR group) in states 2 and 5, where the MAR group has increased but the DRN group decreased connectivity. Univariate ANOVA results in Figure 5 show a MAD group with higher connectivity than the DRN group in state 1, suggesting that alcohol reduced connectivity between postcentral and inferior frontal gyrus, but mixed alcohol and marijuana diminished the effect of alcohol. A similar trend in higher connectivity in MAD vs DRN groups is observed in state 4 . Increased connectivity in MAD compared with DRN groups could in part be explained by reported increments of structural connectivity in the prefrontal cortex linked to marijuana use (Filbey et al, 2014). More evidence can be found comparing the brain of adolescents, where subjects that binge drink and consume marijuana exhibited less white matter alterations than those who only consumed alcohol (Jacobus et al, 2009). Even though a subtractive effect is plausible, the consequences do not translate into beneficial outcomes. Comorbid alcohol and marijuana consumption 
does have a toll in neurocognitive abilities including verbal learning, memory, attention, processing speed, visuospatial functioning, and cognitive control (Squeglia and Gray, 2016). The opposite trend between alcohol and cannabis is not an indication that detrimental neurocognitive effects will diminish because of concurrent use.

The results obtained for combined nicotine and marijuana show connectivity effects that contrast with single substance use. Connectivity strength in state 4 is dramatically increased compared with all of the other samples groups. In states 1 and 6, the connectivity strength of the SAM group is lower than all other groups. These connectivity differences do not follow an obvious trend, nor an additive effect, when compared with the MAR and SMK groups. The chemistry of combined marijuana nicotine use is characterized by an increase of nicotinic acetylcholine receptor ( $\mathrm{nAChR}$ ) availability in the prefrontal cortex and the thalamus as compared with single nicotine use (Brody et al, 2016). In the same work, this interaction thought to occur at the cell molecular level was also found in mixed nicotine caffeine consumption. Availability of nAChRs modulate whole brain connectivity measures such as global network efficiency that measures the efficiency of information transfer through the brain (Wylie et al, 2012). In similar fashion, chemical interactions of nicotine and marijuana may have potentiated the variety of global connectivity strength effects that are seen in Figure $4 \mathrm{~h}$. These outcomes must be interpreted in the context of whole brain analysis and cannot be used to describe more specific effects of each substance. Univariate ANOVA outcomes of state 4 (see Figure 5) are compatible with a difference; specifically, an increment of connectivity in comorbid marijuana and nicotine use as compared with single substance use. However, the SAM group did not show differences with controls, indicating that observed combined $v s$ single use effects are not simple to explain.

Up to this point, we can observe that some states are more affected by certain substances. For example, alcohol has a consistently strong influence in state 2 . Marijuana produced a large increase of connectivity strength in states 1,2 , and 3. Nicotine produced a large effect size between dorsal striatum and sensorimotor areas in state 4 . We can observe that the ALL group influenced by all three substances might follow the trend of one of the three substances on different states. In state 1, ALL and MAR groups had a similar connectivity strength. This increment in the ALL could also be seen in the univariate results for state 1 (shown in Figure 5) and is an opposite effect to the decrease connectivity in the DRN group in that state. If the interaction of marijuana and alcohol can be thought of as additive, then marijuana could have a stronger influence than alcohol in that state. Both multivariate and univariate results agree that alcohol is the stronger influence in state 2 . In Figure $4 \mathrm{~h}$, the ALL group may not have achieved the same decrement of connectivity strength as that seen with the SAD and DRN groups because of the influence of marijuana. It is noteworthy that the MAR group had a very strong increment of connectivity strength. Note that ALL and MAD groups showed similar connectivity strength. The univariate results for state 2 in Figure 5 are consistent with a decrement of connectivity in the DRN and ALL groups, suggesting that alcohol was the most influencing substance. With respect to nicotine, only the univariate results show a consistent similarity between ALL and SMK groups in state 4, suggesting that nicotine was the most influential substance. The effect was strong in specific areas of the brain, dorsal striatum, and sensorimotor areas, but was not observed when analyzing the connectivity strength. We observed effects that were more focused than global effects related to nicotine.

An important limitation of this study was the disparity on the number of samples, where there is a relatively large number of alcohol users, but a low number of marijuana users. Although the low number of marijuana users allowed the observation of effects using MANOVA, the low statistical power was more evident when analyzing single dFNCs. Many univariate results with marijuana effects were excluded after multicomparison correction, but the $P$-values were close to being significant after FDR. Unfortunately, subjects suitable for single marijuana consumption are difficult to find and, along with the need for correcting over a large amount of comparisons, causes a considerable limitation of statistical power. Nevertheless, multivariate analysis picked up strong signals from whole brain marijuana effects because it combined the available information. The MAR group shows similar reduction of occupancy rate with the DRN and MAD subjects in state 2 , but this reduction did not achieve significance mainly because of the small number of subjects in MAR. However, the observed trend is compatible among these three sample groups, indicating that alcohol and cannabis might have the same effects on the occupancy rate of state 2 . In state 4 , the MAR group exhibits an even higher mean occupancy than DRN (group that differ from CTR), but high variability of dFNC from MAR samples decremented detection power. The second important limitation was the lack of covariate measures for the CTR group. Although AUDIT was missing from this group, several studies indicate that AUDIT and DSM-IV have similar specificity (Dawson et al, 2012; Foxcroft et al, 2015), providing evidence that subjects in the CTR group were correctly classified. However, the same cannot be inferred for the other important measures of BID, BAI, ImpSS, and Income. For this reason, linear correlation analysis was limited to the substance user. Third, the small number of single dFNC findings likely reflects only the strongest effects. Other existing effects, such as those observed by hypotheses-driven techniques (Chanraud et al, 2011; Janes et al, 2012), may be missed. The small number within the MAR group plays a role in this limitation.

\section{FUNDING AND DISCLOSURE}

The authors declare no conflict of interest.

\section{ACKNOWLEDGMENTS}

We thank the staff of The Mind Research Network and of the University of Colorado, Boulder, for their assistance with data integration and text editing, in particular Rupa Sabbineni, Brittny Miller, Ellen Blake, Christina Weywadt, and Eric Claus. This work was supported by grants from the National Institutes of Health grant numbers 2R01EB005846, R01REB020407, and P20GM103472 to VDC, R01AA012238 and R01DA030344 to KEH, and National Science Foundation (NSF) grants $1539067 / 1631819$ to VDC. 


\section{REFERENCES}

Allen EA, Damaraju E, Plis SM, Erhardt EB, Eichele T, Calhoun VD (2014). Tracking whole-brain connectivity dynamics in the resting state. Cereb Cortex 24: 663-676.

Allen EA, Erhardt EB, Damaraju E, Gruner W, Segall JM, Silva RF et al (2011). A baseline for the multivariate comparison of restingstate networks. Front Syst Neurosci 5: 2.

Beck AT, Steer RA, Carbin MG (1988). Psychometric properties of the Beck Depression Inventory: twenty-five years of evaluation. Clin Psychol Rev 8: 77-100.

Brody AL, Hubert R, Mamoun MS, Enoki R, Garcia LY, Abraham P et al (2016). Nicotinic acetylcholine receptor availability in cigarette smokers: effect of heavy caffeine or marijuana use. Psychopharmacology 233: 3249-3257.

Brody AL, Olmstead RE, London ED, Farahi J, Meyer JH, Grossman $\mathrm{P}$ et al (2004). Smoking-induced ventral striatum dopamine release. Am J Psychiatry 161: 1211-1218.

Calhoun V, Adali T, Pearlson G, Pekar J (2001). A method for making group inferences from functional MRI data using independent component analysis. Hum Brain Mapp 14: 140-151.

Calhoun VD, Adali T (2012). Multisubject independent component analysis of fMRI: a decade of intrinsic networks, default mode, and neurodiagnostic discovery. IEEE Rev Biomed Eng 5: 60-73.

Camchong J, Stenger A, Fein G (2013a). Resting-state synchrony during early alcohol abstinence can predict subsequent relapse. Cereb Cortex 23: 2086-2099.

Camchong J, Stenger A, Fein G (2013b). Resting-state synchrony in long-term abstinent alcoholics. Alcohol Clin Exp Res 37: 75-85.

Camchong J, Stenger VA, Fein G (2013c). Resting-state synchrony in short-term versus long-term abstinent alcoholics. Alcohol Clin Exp Res 37: 794-803.

Ceballos NA, Tivis R, Lawton-Craddock A, Nixond SJ (2006). Nicotine and cognitive efficiency in alcoholics and illicit stimulant abusers: implications of smoking cessation for substance users in treatment. Subst Use Misuse 41: 265-281.

Chanraud S, Pitel A-L, Pfefferbaum A, Sullivan EV (2011). Disruption of functional connectivity of the default-mode network in alcoholism. Cereb Cortex 21: 2272-2281.

Cheng H, Skosnik PD, Pruce BJ, Brumbaugh MS, Vollmer JM, Fridberg DJ et al (2014). Resting state functional magnetic resonance imaging reveals distinct brain activity in heavy cannabis users - a multi-voxel pattern analysis. J Psychopharmacol 28: $1030-1040$.

Çöl IA, Sönmez MB, Vardar ME, Köşesi H (2016). Evaluation of interoceptive awareness in alcohol-addicted patients. Evaluation 53: $17-22$.

Cousijn J, Wiers RW, Ridderinkhof KR, van den Brink W, Veltman DJ, Goudriaan AE (2012). Grey matter alterations associated with cannabis use: results of a VBM study in heavy cannabis users and healthy controls. Neuroimage 59: 3845-3851.

Craig A (2003). Interoception: the sense of the physiological condition of the body. Curr Opin Neurobiol 13: 500-505.

Dawson DA, Smith SM, Saha TD, Rubinsky AD, Grant BF (2012). Comparative performance of the AUDIT-C in screening for DSM-IV and DSM-5 alcohol use disorders. Drug Alcohol Depend 126: 384-388.

Doyon WM, Thomas AM, Ostroumov A, Dong Y, Dani JA (2013). Potential substrates for nicotine and alcohol interactions: a focus on the mesocorticolimbic dopamine system. Biochem Pharmacol 86: 1181-1193.

Dupuy M, Chanraud S (2016). Imaging the addicted brain: alcohol. Int Rev Neurobiol 129: 1-31.

Everitt BJ, Robbins TW (2005). Neural systems of reinforcement for drug addiction: from actions to habits to compulsion. Nat Neurosci 8: 1481-1489.
Fagerström K-O (1978). Measuring degree of physical dependence to tobacco smoking with reference to individualization of treatment. Addict Behav 3: 235-241.

Filbey FM, Aslan S, Calhoun VD, Spence JS, Damaraju E, Caprihan A et al (2014). Long-term effects of marijuana use on the brain. Proc Natl Acad Sci USA 111: 16913-16918.

First MB, Spitzer RL, Gibbon M, Williams JBW (2002). Structured Clinical Interview for DSM-IV-TR Axis I Disorders, Research Version, Patient Edition. State Psychiatric Institute: New York.

Fisher RA (1937). The Design of Experiments. Oliver and Boyd: Edinburgh; London.

Fox MD, Snyder AZ, Vincent JL, Corbetta M, Van Essen DC, Raichle ME (2005). The human brain is intrinsically organized into dynamic, anticorrelated functional networks. Proce Natl Acad Sci USA 102: 9673-9678.

Foxcroft DR, Smith L, Thomas H, Howcutt S (2015). Accuracy of Alcohol Use Disorders Identification Test (AUDIT) for detecting problem drinking in 18-35 year-olds in England. Viitattu 21: 2015.

Fransson P (2005). Spontaneous low-frequency BOLD signal fluctuations: an fMRI investigation of the resting-state default mode of brain function hypothesis. Hum Brain Mapp 26: 15-29.

Friston KJ (2003). Statistical Parametric Mapping. In: Kötter R (eds). Neuroscience Databases. Springer, Boston, MA.

Ghatan P, Ingvar M, Eriksson L, Stone-Elander S, Serrander M, Ekberg K et al (1998). Cerebral effects of nicotine during cognition in smokers and non-smokers. Psychopharmacology 136: 179-189.

Goldstein RZ, Volkow ND (2002). Drug addiction and its underlying neurobiological basis: neuroimaging evidence for the involvement of the frontal cortex. Am J Psychiatry 159: 1642-1652.

Iatan IF (2010). The Fisher's linear discriminant. Advances in Intelligent and Soft Computing Combining Soft Computing and Statistical Methods in Data Analysis Berlin. Springer-Verlag Berlin: Berlin, Germany, pp 345-352.

Jacobsen LK, Pugh KR, Constable RT, Westerveld M, Mencl WE (2007). Functional correlates of verbal memory deficits emerging during nicotine withdrawal in abstinent adolescent cannabis users. Biol Psychiatry 61: 31-40.

Jacobus J, McQueeny T, Bava S, Schweinsburg BC, Frank LR, Yang TT et al (2009). White matter integrity in adolescents with histories of marijuana use and binge drinking. Neurotoxicol Teratol 31: 349-355.

Janes AC, Nickerson LD, Kaufman MJ (2012). Prefrontal and limbic resting state brain network functional connectivity differs between nicotine-dependent smokers and non-smoking controls. Drug Alcohol Depend 125: 252-259.

Janes AC, Park MT, Farmer S, Chakravarty MM (2015). Striatal morphology is associated with tobacco cigarette craving. Neuropsychopharmacology 40: 406-411.

Jansen JM, van Holst RJ, van den Brink W, Veltman DJ, Caan MW, Goudriaan AE (2015). Brain function during cognitive flexibility and white matter integrity in alcohol-dependent patients, problematic drinkers and healthy controls. Addict Biol 20: 979-989.

Jasinska AJ, Zorick T, Brody AL, Stein EA (2014). Dual role of nicotine in addiction and cognition: a review of neuroimaging studies in humans. Neuropharmacology 84: 111-122.

Koob GF, Volkow ND (2010). Neurocircuitry of addiction. Neuropsychopharmacology 35: 217-238.

Kril JJ, Halliday GM, Svoboda MD, Cartwright H (1997). The cerebral cortex is damaged in chronic alcoholics. Neuroscience 79: 983-998.

Lawrence NS, Ross TJ, Stein EA (2002). Cognitive mechanisms of nicotine on visual attention. Neuron 36: 539-548.

Leshner AI (1997). Addiction is a brain disease, and it matters. Science 278: 45-47. 
Leyfer OT, Ruberg JL, Woodruff-Borden J (2006). Examination of the utility of the Beck Anxiety Inventory and its factors as a screener for anxiety disorders. J Anxiety Disord 20: 444-458.

Lloyd S (1982). Least squares quantization in PCM. IEEE Trans Inform Theory 28: 129-137.

Louie K, Wilson MA (2001). Temporally structured replay of awake hippocampal ensemble activity during rapid eye movement sleep. Neuron 29: 145-156.

Lynall ME, Bassett DS, Kerwin R, McKenna PJ, Kitzbichler M, Muller U et al (2010). Functional connectivity and brain networks in schizophrenia. J Neurosci 30: 9477-9487.

Medina KL, McQueeny T, Nagel BJ, Hanson KL, Yang TT, Tapert SF (2009). Prefrontal cortex morphometry in abstinent adolescent marijuana users: subtle gender effects. Addict Biol 14: 457-468.

Medina KL, Nagel BJ, Tapert SF (2010). Abnormal cerebellar morphometry in abstinent adolescent marijuana users. Psychiatry Res 182: 152-159.

Meyerhoff DJ, Tizabi Y, Staley JK, Durazzo TC, Glass JM, Nixon SJ (2006). Smoking comorbidity in alcoholism: neurobiological and neurocognitive consequences. Alcohol Clin Exp Res 30: 253-264.

Moore BL, Schneider JA, Ryan JJ (1987). Fagerstrom's tolerance questionnaire: clarification of item and scoring ambiguities. Addict Behav 12: 67-68.

Nestler EJ (2005). Is there a common molecular pathway for addiction? Nat Neurosci 8: 1445-1449.

Okita K, Mandelkern MA, London ED (2016). Cigarette use and striatal dopamine D2/3 receptors: possible role in the link between smoking and nicotine dependence. Int J Neuropsychopharmacol 19: 1-5.

Pariyadath V, Gowin JL, Stein EA (2016). Resting state functional connectivity analysis for addiction medicine: From individual loci to complex networks. Prog Brain Res 224: 155-173.

Pariyadath V, Stein EA, Ross TJ (2014). Machine learning classification of resting state functional connectivity predicts smoking status. Front Hum Neurosci 8: 425.

Pujol J, Blanco-Hinojo L, Batalla A, Lopez-Sola M, Harrison BJ, Soriano-Mas C et al (2014). Functional connectivity alterations in brain networks relevant to self-awareness in chronic cannabis users. J Psychiatr Res 51: 68-78.

Rachakonda S, Egolf E, Correa N, Calhoun V (2007). Group ICA of fMRI toolbox (GIFT) manual. https://www.nitrc.org/docman/view. php/55/295/v1_203d_GIFTManual.pdf (cited 5 November 2011).

Richmond-Rakerd LS, Slutske WS, Lynskey MT, Agrawal A, Madden PA, Bucholz KK et al (2016). Age at first use and later substance use disorder: shared genetic and environmental pathways for nicotine, alcohol, and cannabis. J Abnorm Psychol 125: 946.

Saunders JB, Aasland OG, Babor TF, Grant M (1993). Development of the alcohol use disorders identification test (AUDIT): WHO collaborative project on early detection of persons with harmful alcohol consumption-II. Addiction 88: 791-804.

Sjoerds Z, Stufflebeam SM, Veltman DJ, Van den Brink W, Penninx BW, Douw L (2017). Loss of brain graph network efficiency in alcohol dependence. Addict Biol 22: 523-534.

Squeglia LM, Gray KM (2016). Alcohol and drug use and the developing brain. Curr Psychiatry Rep 18: 46.
Subramaniyan M, Dani JA (2015). Dopaminergic and cholinergic learning mechanisms in nicotine addiction. Ann NY Acad Sci 1349: 46-63.

Sutherland MT, McHugh MJ, Pariyadath V, Stein EA (2012). Resting state functional connectivity in addiction: Lessons learned and a road ahead. Neuroimage 62: 2281-2295.

Sweitzer MM, Geier CF, Addicott MA, Denlinger R, Raiff BR, Dallery J et al (2016). Smoking abstinence-induced changes in resting state functional connectivity with ventral striatum predict lapse during a quit attempt. Neuropsychopharmacology 41: 2521-2529.

Tiffany ST, Conklin CA (2000). A cognitive processing model of alcohol craving and compulsive alcohol use. Addiction 95: 145-153.

Verdejo-Garcia A, Clark L, Dunn BD (2012). The role of interoception in addiction: a critical review. Neurosci Biobehav Rev 36: 1857-1869.

Vergara VM, Liu J, Claus ED, Hutchison K, Calhoun V (2017a). Alterations of resting state functional network connectivity in the brain of nicotine and alcohol users. Neuroimage 151: 45-54.

Vergara VM, Mayer AR, Damaraju E, Hutchison K, Calhoun VD (2017b). The effect of preprocessing pipelines in subject classification and detection of abnormal resting state functional network connectivity using group ICA. Neuroimage 145(Pt B): 365-376.

Volkow ND, Baler RD, Compton WM, Weiss SR (2014). Adverse health effects of marijuana use. N Engl J Med 370: 2219-2227.

Warne RT (2014). A primer on multivariate analysis of variance (MANOVA) for behavioral scientists. Pract Assess Res Eval 19.

Weiland BJ, Sabbineni A, Calhoun VD, Welsh RC, Bryan AD, Jung $\mathrm{RE}$ et al (2014). Reduced left executive control network functional connectivity is associated with alcohol use disorders. Alcohol Clin Exp Res 38: 2445-2453.

Williams LJ, Abdi H (2010). Fisher's least significant difference (LSD) test. Salkind NJ, Dougherty DM, Frey B (eds). Encyclopedia of Research Design. SAGE Publications, Inc.: Thousand Oaks, CA, 218: 840-853.

Winstock AR (2014). The global drug survey 2014. findings. Global Drug Survey; http://www.globaldrugsurvey.com/facts-figures/theglobal-drug-survey-2014-findings/.

Wise RA (2008). Dopamine and reward: the anhedonia hypothesis 30 years on. Neurotox Res 14: 169-183.

Wise RA (2010). Neuroleptics and operant behavior: the anhedonia hypothesis. Behav Brain Sci 5: 39.

Wylie KP, Rojas DC, Tanabe J, Martin LF, Tregellas JR (2012). Nicotine increases brain functional network efficiency. Neuroimage 63: 73-80.

Yeh PH, Simpson K, Durazzo TC, Gazdzinski S, Meyerhoff DJ (2009). Tract-Based Spatial Statistics (TBSS) of diffusion tensor imaging data in alcohol dependence: abnormalities of the motivational neurocircuitry. Psychiatry Res 173: 22-30.

Zalesky A, Solowij N, Yucel M, Lubman DI, Takagi M, Harding IH et al (2012). Effect of long-term cannabis use on axonal fibre connectivity. Brain 135(Pt 7): 2245-2255.

Zuckerman M (1996). Item revisions in the sensation seeking scale form V (SSS-V). Personality and Individual Differences 20: 515.

Supplementary Information accompanies the paper on the Neuropsychopharmacology website (http://www.nature.com/npp) 